\title{
On how adults identify the orientation of a shape
}

\author{
LILA GHENT BRAINE, LINDA RELYEA, and LYNN DAVIDMAN \\ Barnard College, Columbia University, New York, New York 10027
}

\begin{abstract}
It is generally assumed that the orientation of a shape is judged only by identifying the location of a particular feature. In contrast, it is argued here that the basis of orientation judgments depends on the task; this proposal is examined for judgments of upside down and sideways. Pictures of mono-oriented objects were presented one at a time, and adults identified the orientations of each in a RT paradigm. One condition presented successively upright and upside-down pictures or upright and sideways pictures. Another condition presented successively upside-down and sideways pictures. As predicted, the time to identify orientation was longer for the condition presenting two nonuprights, because specific identifications of upside down and sideways were required, whereas the other condition required only that uprightness be distinguished from nonuprightness. The notion that the nature of a judgment of shape orientation depends on the contrasts provided by the task has implications for theory and experimental design that are discussed for both realistic and geometric shapes.
\end{abstract}

A judgment of the orientation of a shape is ordinarily thought to describe the relation between a particular feature of the shape, usually the top, and a spatial framework. From this point of view, all judgments of orientation are based on essentially the same underlying processes. If it is more difficult to identify a shape as turned to the left than as upright, this difference is attributed to a difference in the ease of identifying the top and left positions of space. Consequently, work in the area has dealt with the role of different frameworks in judgments of shape orientation (reviewed by Howard \& Templeton, 1966, pp. 322-331) and in recognition of shapes (Rock, 1974) or, more recently, with judgments of spatial location (Just \& Carpenter, 1975; Maki, Maki, \& Marsh, 1977) and memory for spatial locations (Mandler \& Parker, 1976).

There have been few attempts to investigate directly how adults identify the orientation of a shape. The few available studies (Butler, 1964; Farrell, 1979; Maki, 1979; Maki, Grandy, \& Hauge, 1979) have focused on comparing the speed of identifying updown and left-right orientations of geometric shapes under various conditions, and, in general, have found that up-down judgments require less time than leftright judgments (Note 1). Because a judgment of the orientation of a shape has been conceived of simply as a problem in identifying the location of a particular feature, these studies have been limited in at least

This research was supported by Grant HD 09513 from the National Institutes of Health. The authors are grateful for two kinds of help: the testing of some subjects by Catherine Lerner, and constructive criticism by two anonymous reviewers and especially by Martha Teghtsoonian. Requests for reprints should be sent to Lila G. Braine, Department of Psychology, Barnard College, Columbia University, New York, New York 10027. two ways. First, only a few simple shapes have served as stimuli-an arrow, a T-shape, and a V-shape. The population of shapes from which this sample is drawn is very narrow indeed; the shapes consist of two to three lines with one intersection, symmetrical about one axis, and probably characterized by intrinsic directionality (Howard \& Templeton, 1966, pp. 295301). Second, the work has not investigated commonly used judgments of orientation such as upright or upside down. It is not to be taken for granted that judging an arbitrarily specified feature as being at the top of a shape is the same as judging a cat as appearing upright.

The present study broadens the scope of the inquiry into the perception of shape orientation in several ways. The stimuli consist of pictures of objects with a usual upright, a range of pictures is used and the judgments of upright, upside down, and sideways are explicitly investigated. The theoretical basis for this work challenges the assumption that all judgments of shape orientation are made by describing the location of a particular feature and assumes, instead, that orientation information may be processed in different ways that vary in complexity (Braine, 1978b). The general approach will be described in terms that clearly apply to realistic shapes, and the basis for including geometric shapes will be presented in the discussion.

The simplest orientation judgment is one that identifies a shape as upright or nonupright. How might such a judgment be made? Let us assume that shapes are represented in memory in the phenomenal upright, an assumption that has already been made for letters (e.g., Cooper \& Shepard, 1973), for objects that have a usual orientation in the environment (Rock, 1966, p. 34), and for shapes in general (Braine, 1973). The orientation of a shape could be identified as upright or nonupright on the basis of whether the 
input matched or did not match the orientation of the internal representation (Braine, 1978b; Braine \& Knox, 1975 , p. 478). The perception of a match or mismatch is assumed to occur automatically as part of the identification of a shape; for example, a car is not identified simply as a car, but necessarily as an upright or nonupright car. This global judgment does not provide a basis for distinguishing among nonupright shapes: Upside down is not discriminated from sideways nor is left-turned discriminated from rightturned, but each of these nonupright orientations is equally discriminable from upright.

Additional cognitive processing is required to identify the orientation of a nonupright shape as specifically upside down or sideways. The distinction between these two nonuprights could be based on the perceived verticality or horizontality of the axis joining the top and bottom features of the shape. That is, a nonupright shape in which the top-bottom axis is seen as vertical is identified as upside down and a nonupright in which the top-bottom axis is horizontal is identified as sideways. The amount of processing that permits the identification of turned-to-the-side does not, however, provide the basis for identifying a sideways shape as turned specifically left or right.

To identify a shape as left-turned or right-turned, a feature of the shape must be related to the sides of a spatial framework, provided either by the body or by the environment. Left and right are inherently relational judgments in the sense that the position of the shape must be described with respect to something else, whereas, as suggested above, some orientation judgments may be made without describing the relation between the shape and another feature of the environment. From this point of view, it is the greater cognitive complexity of this relational judgment that makes left-right identifications more difficult than up-down identifications. The more traditional view, by contrast, assumes that the same cognitive processes underlie left-right and up-down identification, and attributes the greater difficulty of leftright tasks to the less differentiated character of the horizontal axis relative to the vertical axis of space (e.g., Farrell, 1979).

Several studies with young children provide support for the distinctions proposed, in particular, for the distinction between identifying upside-down and sideways stimuli as nonupright shapes and as specifically upside down and sideways. Fagan and Shepherd (1979) found that infants discriminated upright faces from upside-down and sideways faces before they discriminated upside-down and sideways faces from each other, and the latter discrimination preceded a discrimination between faces rotated 90 deg left or right. Both a study with 2-year-old children (Braine, 1978a) and a subsequent investigation with children of 3 and 4 years (Braine, Lerner, \& Relyea, 1980) found that upright and nonupright shapes were distinguished from each other far more easily than nonuprights (upside down and sideways) were discriminated from each other. The present study asks whether analogous differences in the processing of orientation can be found in adults.

Essentially, adults were asked to identify the orientations of pictures of familiar objects with a customary upright (such as a house or a dog), and the time taken to make the judgments was measured. The pictures were presented under two conditions. In one condition, nonupright pictures (upside down for half the observers and sideways for the other half) were presented one at a time in a block of trials mixed with upright pictures; in the other condition, the upsidedown and sideways pictures were presented one at a time in the same block of trials. Since the identifications of upright and nonupright orientations are assumed to require less cognitive processing than do judgments of the identification of nonuprights as specifically upside down or sideways, the reaction times for the blocks containing the upright and nonupright pictures should be faster than for the blocks containing two nonuprights. In other words, the identification of a picture as upside down (or sideways) should take less time in the block with upright pictures than in the block with nonupright pictures. On the other hand, the traditional view of how orientation judgments are made would predict that the time to identify a single picture as upside down or sideways would be unaffected by other orientations presented in the same block of trials: Regardless of condition, a subject would judge a figure as upside down when the figure was oriented with the top feature at the bottom and as sideways when the figure was oriented with the top feature at the side.

\section{METHOD}

\section{Subjects}

Seventy-two right-handed college students participated in the study. The $\mathbf{4 8}$ females and 24 males were drawn primarily from introductory psychology, with a small number of volunteers from other groups of students. Data from 10 other students were discarded because of long reaction times; the pattern of results for these subjects was the same as that for other subjects.

\section{Apparatus and Stimuli}

The stimuli were black line drawings of 20 familiar, monooriented shapes. The asymmetrical, and essentially side-view, shapes were bicycle, car, cat, dog, elephant, horse, kettle, sailboat, teacup, tricycle, and two trucks. The roughly symmetrical, and essentially front-view, shapes were boy, face, flowers, girl, house, lamp, table, and tree. The long axes of the drawings averaged $2.1 \mathrm{~cm}$, with a range of 1.5 to $2.5 \mathrm{~cm}$, and the drawings were presented in the center of a square aperture, $6.25 \mathrm{~cm}$ on a side. The drawings could appear in any one of three orientations: upright, upside down (rotated $180 \mathrm{deg}$ in the picture plane), or sideways (rotated 90 deg counterclock wise).

The pictures were presented in a Scientific Prototype tachistoscope (Model 800F) in which the distance between the subjects' 
eyes and the stimulus was $80 \mathrm{~cm}$. The exposure duration was $250 \mathrm{msec}$ at maximum intensity; the blank interstimulus interval was illuminated at a medium intensity judged by the experimenters to yield minimum contrast between the exposure field and the blank field. The beginning of each stimulus presentation triggered a Hunter Klockounter (Model 120C), which stopped when the observer pressed a response button indicating the perceived orientation of the stimulus. The two small buttons were housed side by side and $4.4 \mathrm{~cm}$ apart on the surface of a small box, $12.5 \mathrm{~cm}$ long $\times$ $6.2 \mathrm{~cm}$ wide $\times 3.8 \mathrm{~cm}$ high. The box was held by both hands, one at each end, and the subject pressed the buttons with the thumbs.

\section{Procedure}

The subject was told that he or she would be presented with line drawings of familiar figures in two orientations and that the task was to signal the figure's orientation by pushing a button as quickly and accurately as possible. For the two orientations, one group (upside-down problem) received upside-down and upright pictures in one condition and upside-down and sideways pictures under the other condition. Another group (sideways problem) received sideways and upright pictures under one condition and sideways and upside-down pictures under the other. The words used to describe the orientations to the subjects were "upright," "upside down," and "sideways." A sheet containing the 20 figures to be used was initially presented for inspection and rotated into the two orientations used in the first condition.

At the beginning of a trial, the subject was asked to look at the small dot in the center of the otherwise blank field. A single figure was presented, and the subject responded by pushing a button: After stimulus termination, the blank field with the fixation point reappeared. Each of the 20 figures was presented in the two orientations, making a total of $\mathbf{4 0}$ different stimuli for any condition. However, the first 10 trials of each task were treated as practice trials and were repeated after the 40th trial, resulting in the presentation of 50 trials per condition, the last 40 of which were used for analysis. If a subject made an incorrect response, the stimulus was presented again after the 50th trial without informing her or him that it would be repeated. After the first condition was completed, the subject rested for a few minutes.

The second condition was the same as the first in all respects except that one of the two orientations was changed, as described above. For the group presented with the upside-down problem, the same hand signaled upside down in the two conditions, while the other hand signaled upright in one condition and sideways in the other. For the group receiving the sideways problem, the same hand signaled sideways in the two conditions, while the other hand signaled upright in one condition and upside down in the other.

The order of the conditions (whether a nonupright and an upright, or two nonuprights) was balanced across subjects within each problem. The left and right hands (or buttons) used to signal orientations were balanced across the orientations being presented. The stimuli were presented to each subject in a different random order with the following constraints: (1) The same figure did not appear on two consecutive trials, (2) the same orientation did not appear on more than three consecutive trials, and (3) the two orientations appeared with equal frequency in the first and second halves of the task.

\section{RESULTS}

The main finding was that the identification of upside-down and sideways orientations required, in general, more time when they appeared in the same block of trials than when each appeared in a block with upright stimuli. There was some tendency for upright orientations to be identified most quickly and upside-down orientations to be identified more quickly than sideways. The results will be presented separately for the upside-down and sideways problems because the details differ in some aspects.

For the upside-down problem, the mean reaction times (RTs) for each orientation for each condition are shown in Figure 1, separately for each hand group. The data were analyzed with a three-way, mixed analysis of variance in which the hand used to signal upside down was the between-groups variable; the within-groups variables were the two conditions of presentation and the two orientations of the stimuli, categorized as upside down and not upside down for the purposes of the analysis. Condition exerted a significant effect $[F(1,34)=6.48, p<.02]$, with the mean RT for the upside-down/upright condition being $563 \mathrm{msec}$ and for the upside-down/sideways condition being $603 \mathrm{msec}$. However, this main effect was due primarily to the significant interaction of Hand Group by Condition $[F(1,34)=6.84, p<.02]$; hand group also interacted significantly with orientation $[F(1,34)=6.02, p<.02]$. There were no other significant interactions.

The hand used to signal orientation influenced performance in the following ways. When the left hand signaled upside down and the right hand signaled either upright or sideways, the mean RT for the upsidedown/upright condition was $529 \mathrm{msec}$ and for the upside-down/sideways condition was $609 \mathrm{msec}$, as can be seen in Figure 1. The mean number of errors was 1.9 for the upside-down/upright conditions and 2.2 for the upside-down/sideways conditions, indicating that the difference in RTs between the two conditions could not be accounted for as a tradeoff

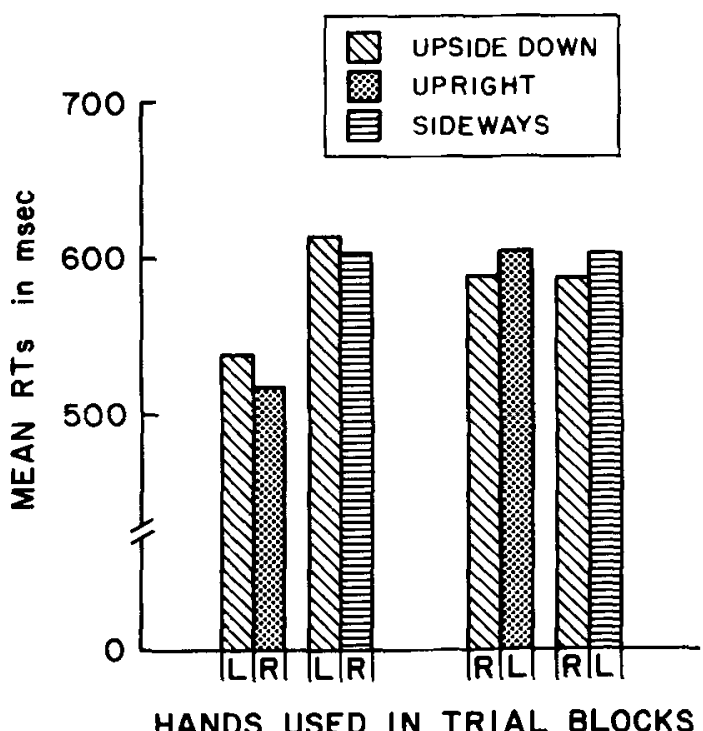

Figure 1. Mean RTs to identify orientation by the two hand groups given the upside-down problem. 
between speed and accuracy. When the right hand signaled upside-down, and the left hand either upright or sideways, the mean RT for the upside-down/ upright condition was $597 \mathrm{msec}$ (mean errors $=1.8$ ) and for the upside-down/sideways condition, $596 \mathrm{msec}$ (mean errors $=2.2$ ). The similarity in performance under the two conditions is due to the relatively long RTs for the subjects signaling upside down with their right hands and upright with their left hands. That is, a response incompatibility appeared when right-handed people were required to signal the wrongly-turnedlooking picture with the right hand and the rightlyturned-looking picture with the left hand. The interaction between hand group and orientation also appears to be due to the long RTs to the upright stimuli of the group signaling upright with their left hands.

For the sideways problem, the mean RT for each orientation for each condition is shown in Figure 2, separately for the two hand groups to permit comparison with the analogous data in Figure 1. The data were analyzed with a three-way, mixed analysis of variance identical to that used for the analysis of the upside-down problem. In the sideways problem, significant effects were exerted by condition $[F(1,34)$ $=18.35, p<.01]$ and by orientation $[F(1,34)=19.53$, $p<.01]$; none of the interactions reached a significant level. Reaction times were significantly faster under the sideways/upright condition ( $532 \mathrm{msec}$ ) than under the sideways/upside-down condition (602 msec). The lower RT was associated with a slightly lower mean error score, 1.5 for the sideways/upright conditions and 1.9 for the sideways/upside-down condi-

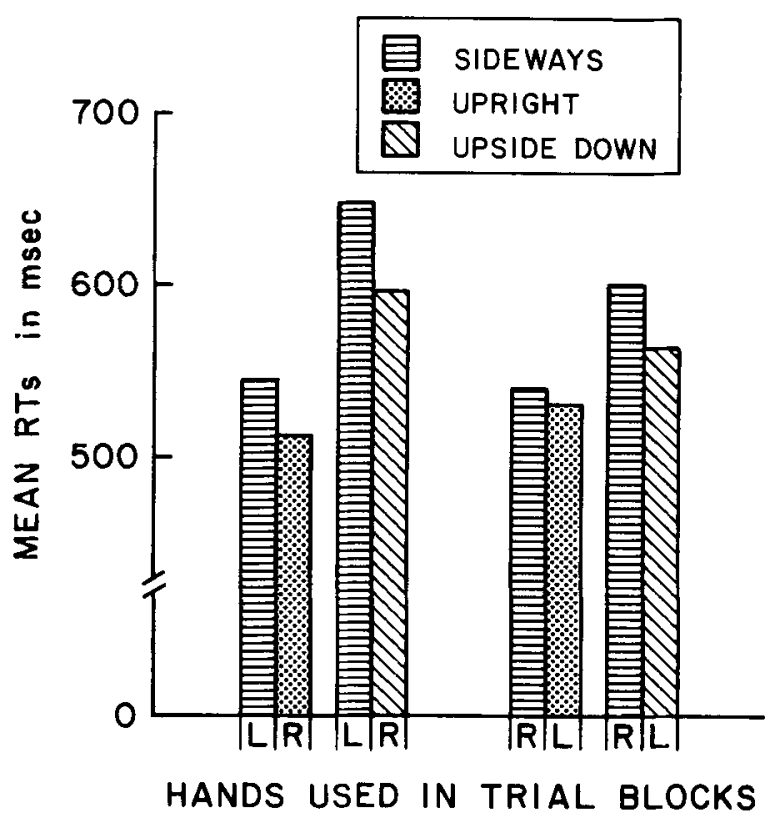

Figure 2. Mean RTs to identify orientation by the two hand groups given the sideways problem. tions. In contrast with the previous problem, the hand used to signal upright and sideways did not have a significant effect on RTs.

The significant effect exerted by orientation in the sideways problem was due to the consistently longer RTs to identify sideways than the not-sideways category comprising upright and upside-down stimuli (see Figure 2). Errors averaged 1.6 for identifying sideways and 1.8 for not-sideways, a difference in the direction of a speed-accuracy tradeoff, in contrast with the paralleling of errors and RTs in the comparisons between conditions. The more rapid identification of not-sideways had two sources. There was a tendency for the upright orientations to be identified slightly more rapidly than the nonupright orientations in both the sideways and the upside-down problems (except for the response incompatibility group). Most of the orientation effect, however, was due to the more rapid identification of upside down than sideways (see Figure 2). Since a comparable effect had not appeared in the upside-down problem using identical stimuli, the effect of order of conditions was examined to see if prior experience in identifying sideways (in the sideways-upright condition) affected the RTs to upside down and sideways. However, order had no effect other than generally decreasing RTs for the condition presented second.

To put the effects of different factors in perspective, the absolute magnitudes of the effects of condition and of orientation were calculated, combining the data across problems. The difference between the upright/nonupright and the nonupright/nonupright conditions was $55 \mathrm{msec}$ for all hand groups combined (and $73 \mathrm{msec}$ when the group showing a response incompatibility was omitted). The difference between upside down and sideways, when all the conditions containing only those orientations were combined, was $23 \mathrm{msec}$. The difference between the upright and nonupright stimuli presented in the same block of trials was $11 \mathrm{msec}$ (and $21 \mathrm{msec}$ when the response incompatibility group was omitted). Condition clearly exerted the major effect on the time taken to identify orientation under the conditions of the present experiment.

\section{DISCUSSION}

The effect of condition on the time taken to identify orientation supports the view that orientation information may be processed to different degrees of specificity; upside down and sideways are identified as nonupright under some conditions and as specifically upside down and sideways under other conditions. The notion that shape orientation may be judged in more than one way contrasts sharply with the prevailing view that all orientation judgments are made on the same basis (i.e., by judging the location of a 
particular feature of the shape). The discussion will deal with two topics-alternative interpretations of the results, and a consideration of the work using geometric shapes from the perspective of the present paper.

\section{Interpretations of the Data}

The possibility that RT differences stem from the magnitudes of the angular differences to be discriminated is an interpretation that can be ruled out. Comparisons among the absolute levels of RTs in Figures 1 and 2 indicate that angular differences played virtually no role in determining RTs. For the hand groups in which the left hand signaled nonupright in the nonupright/upright conditions, RTs were almost identical for the upside-down problem $(529 \mathrm{msec})$ and the sideways problem $(530 \mathrm{msec})$, despite the fact that the pictures differed by $180 \mathrm{deg}$ in the former problem and by $90 \mathrm{deg}$ in the latter problem. For the other hand group in the nonupright/upright condition, much longer RTs occurred for the upside-down problem (because of the response incompatibility discussed earlier) than for the sideways problem, although an angular-difference hypothesis would predict the opposite relation between the tasks. Finally, the sideways pictures differed from both upright and upside down by $90 \mathrm{deg}$, yet sideways was identified significantly more quickly in the context of upright, than in the context of upside-down, pictures.

Another interpretation of the effect of condition on RT is that uprightness was identified more quickly than other orientations (for unknown reasons), and the rapid identification of nonupright orientations was a by-product of the rapid identification of uprightness. This view implies that the subject processed only uprightness and required the same amount of time to classify an item as having this attribute as to classify an item as not having it. Although possible, such an interpretation is inconsistent with the usual result that looking for an attribute (in a simple display), not finding it, and judging it absent requires more time than looking for an attribute, finding it, and judging it present (Clark, Carpenter, \& Just, 1973). However, perhaps the more important point to consider is what is implied by the different interpretations, since there is a sense in which the difference between the two views can be construed as primarily a matter of terminology. The crux of the view underlying the present work is that subjects can respond to the orientation of a single upside-down (sideways) picture in more than one way: as specifically upside down (sideways) or as nonupright. As long as the alternative interpretation acknowledges that subjects must, at some point, classify an upside-down (sideways) stimulus as nonupright, the difference between the views becomes a question of whether the category for the nonspecific orientation should be defined as an absence of uprightness or as looking wrongly turned. The important point is that a new category is required to describe a nonspecific judgment of the orientation of a nonupright shape.

In addition to the effect of condition, two differences in the speed of making orientation judgments appeared that were suggestive, but not conclusive. Upside down was judged more quickly than sideways, but this finding appeared only for the sideways problem. The orientation of upright stimuli tended to be identified slightly more rapidly than nonupright stimuli. However, this effect could reflect a more rapid identification of upright (than nonupright) shapesrather than a more rapid identification of orientationsince upright shapes are usually recognized more easily than nonupright shapes (reviewed by Howard \& Templeton, 1966, pp. 312-322; Kolers \& Perkins, 1969; Rock, 1974).

\section{Geometric Shapes}

The present work has used only shapes of objects that have a customary upright; previous work with adults has used only geometric shapes. Does the line of thought presented here apply only to familiar mono-oriented shapes? There are at least two reasons for believing that the answer is "no." Geometric shapes are generally thought of as neutral with respect to orientation, in the sense that there is no one position that is perceived as upright. However, there is considerable evidence to the contrary, showing that many geometric shapes have an intrinsic top and an apparent upright for adults as well as children (e.g., Ghent, 1961; Howard \& Templeton, 1966, pp. 295301; Schaller \& Harris, 1975). A second, and more fundamental, reason for considering that a geometric shape may be seen as upright, upside down, sideways, and so on, stems from Rock's (1974) proposal that a geometric shape is described or encoded as having a top, bottom, and sides. In Rock's work, the feature at the top of the phenomenal framework when the shape was first presented was coded as the top of the shape. The very notion that geometric shapes are seen as having a top, either intrinsic or assigned arbitrarily by the conditions of presentation, implies that geometric shapes must be referred to as having an apparent upright for any given set of presentation conditions. The different kinds of judgments proposed for mono-oriented realistic shapes obviously apply to geometric shapes as well.

The next question is whether the available studies with geometric shapes provide data relevant to the theory presented here. Unfortunately, the studies are problematic in various ways. Butler's (1964) study, of which the up-down and left-right comparison was a small part, presented only the contrasts of up-withdown stimuli and left-with-right stimuli. This procedure, also used in some of Farrell's (1979) experi- 
ments, essentially turns the up-down task into an upright/nonupright task without providing additional data on the time to identify "down" in the presence of another nonupright. In the work of Maki and her colleagues (Maki, 1979; Maki et al., 1979), up, down, left, and right usually appeared in the same block of trials, but the results presented only the combined up-down means and the combined leftright means.

Two of Farrell's experiments presented up, down, left, and right stimuli in the same block of trials, and provided the times taken to identify each orientation. This work, however, exemplifies another problem (which also appears in the other studies cited). The only stimulus presented was an arrow, which made it possible to treat the four-choice task as two sets of two-choice tasks. That is, the items with a long vertical axis could be identified on an upright/nonupright basis, and the items with a long horizontal axis could be identified as left and right. There has not been concern expressed in the literature about the confounding of left and right orientations with a long horizontal axis and up and down with a long vertical axis. However, identifying a shape as upside down, in contrast with sideways, means that the top-bottom axis is perceived as vertical rather than horizontal; this discrimination is unnecessary when the top-bottom axis is confounded with the long axis of the figure. The lack of attention to this confound may be due to the traditional conception of the problem of identifying orientation as one of identifying the ends of the horizontal and vertical axes.

Farrell noted a tendency for the orientation of the up arrows to be identified more quickly than those of the down arrows, and proposed that the up-down difference stemmed from the asymmetry of the perceived vertical dimension. The logic of the argument is dubious, since an asymmetry would presumably permit the ends to be identified easily but would provide no basis for expecting one end to be identified more easily than the other. An alternative explanation is that the orientation of the up arrows was identified more readily (in a two-choice task) on the same basis that the orientation of the upright realistic figures showed a tendency to be identified more readily. Assume that the up arrow was perceived to be in the upright or normative position. A normative position could be identified more quickly either because this orientation matched that of the encoded shape or because the shape itself was identified more quickly in the upright position (as already suggested in the discussion of the realistic shapes).

\section{Conclusions}

The point of view presented here has implications for both theory and experimental procedures in the area of orientation perception. The usual assumption that the orientation of a shape is judged in one way, by describing the location of a particular feature of the shape, needs to be reassessed. The present results suggest that the orientation of a single, nonupright shape can be judged at varying degrees of specificity, depending on the task demands. In principle, the categorical judgments useful for realistic shapes are also appropriate for describing the orientation of geometric shapes. A second assumption to be questioned is that upright and upside-down orientations are identified more easily than left and right orientations because the poles of the vertical axis are more discriminable than those of the horizontal axis. An alternative consistent with the results here is that the differences are due to the different amounts of cognitive processing required to make the various judgments.

Some new procedural issues have been raised by the present work. The common practice of pairing upright with upside-down stimuli does not provide a valid measure of the difficulty of identifying the orientation of upside down. To put the issue more generally, the specificity of any orientation judgment will depend on the orientation contrasts provided by the task. Furthermore, the characteristics of the stimuli presented require consideration. To investigate judgments of the orientation of shapes, the top-bottom axis of the shape cannot be confounded with the long axis, and asymmetrical as well as symmetrical shapes should be used to eliminate the possibility that orientation information is processed in special ways with symmetrical figures (since symmetry is more readily perceived about the vertical than about the horizontal axis). Finally, the use of familiar, mono-oriented shapes rather than nonsense shapes may clarify the nature of the stimulus attributes used in judging shape orientation; the use of meaningful verbal material in learning tasks certainly revealed responsiveness to stimulus attributes not readily tapped by earlier work with nonsense syllables.

\section{REFERENCES}

Braine, L. G. Perceiving and copying the orientation of geometric shapes. Journal of Research and Development in Education, 1973, 6, 44-55.

Braine, L. G. Early stages in the perception of orientation. In M. Bortner (Ed.), Cognitive development and growth. New York: Brunner/Mazel, 1978. (a)

Braine, L. G. A new slant on orientation perception. American Psychologist, 1978, 33, 10-20. (b)

Braine, L. G., \& Knox, C. Children's orientation judgments: Retinally or environmentally determined? Perception \& Psychophysics, 1975, 17, 473-479.

Braine, L. G., Lerner, C., \& Relyea, L. Levels in the identifying of orientation by preschool children. Journal of Experimental Child Psychology, 1980, 30, 171-185.

BUTLER, J. Visual discrimination of shape by humans. Quarterly Journal of Experimental Psychology, 1964, 16, 272-276.

Clakk, H. H., Carpenter, P. A., \& Just, M. A. On the meeting of semantics and perception. In W. G. Chase (Ed.), Visual information processing. New York: Academic Press, 1973. 
Cooper, L. A., \& Shepard, R. N. Chronometric studies of the rotation of mental images. In W. G. Chase (Ed.), Visual information processing. New York: Academic Press, 1973.

FAGAN, J. F., \& ShePhERd, P. A. Infants' perception of face orientation. Infant Behavior and Development, 1979, 2, 227-234.

FARRELl, W. S., JR. Coding left and right. Journal of Experimental Psychology: Human Perception and Performance, 1979, 5, $42-51$.

Ghent, L. Form and its orientation: A child's-eye view. American Journal of Psychology, 1961, 74, 177-190.

Howard, I. P., \& Templeton, W. G. Human spatial orientation. New York: Wiley, 1966.

Just, M. A., \& Carpenter, P. A. The semantics of locative information in pictures and mental images. British Journal of Psychology, 1975, 66, 427-441.

Kolers, P. A., \& Perkins, D. N. Orientation of letters and errors in their recognition. Perception \& Psychophysics, 1969, 5, 265-269.

MAKI, R. H. Right-left and up-down are equally discriminable in the absence of directional words. Bulletin of the Psychonomic Society, 1979, 14, 181-184.

Makı, R. H., Grandy, C. A., \& Hauge, G. Why is telling right from left more difficult than telling above from below? Journal of Experimental Psychology: Human Perception and Performance, $1979,5,52-67$.

MaKı, R. H., MAKI, W. S., \& MarSh, L. G. Processing locational and orientational information. Memory \& Cognition, 1977, 5, 602-612.

Mandler, J. M., \& Parker, R. E. Memory for descriptive and spatial information in complex pictures. Journal of Experimental Psychology: Human Learning and Memory, 1976, 2, 38-48.

Rock, I. The nature of perceptual adaptation. New York: Basic Books, 1966.

Rock, I. Orientation and form. New York: Academic Press, 1974.
Schaller, M. J., \& Harris, L. G. "Upright" orientations of forms change with subject age and with features of form. Perception \& Psychophysics, 1975, 17, 179-188.

Staller, J., \& Sekuler, R. Mirror-image confusion in adults and children. American Journal of Psychology, 1976, 89, 253-268.

WolfF, P. Mirror-image confusability in adults. Journal of Experimental Psychology, 1971, 91, 268-272.

\section{NOTE}

1. Two sets of data seem to conflict with this conclusion. One set involves same-difference judgments of two figures simultaneously presented in different configurations (Staller \& Sekuler, 1976; Wolff, 1971). Because such judgments need not identify how a particular figure is turned (but only whether two figures are turned the same way), these studies are not considered in the present paper. The second set of data shows that up-down and left-right differences do not appear when the positions of a shape are designated by letters (Maki, 1979; Maki et al., 1979). The question is whether the subjects were making spatial judgments when using letters or whether they used the letters to identify four different shapes: a horizontal line with an arrowhead at the left, a vertical line with an arrowhead at the top, a horizontal line with an arrowhead at the right, and a vertical line with an arrowhead at the bottom. Although the data do not permit a resolution of the issue, it is at least as likely that the subjects used the letters to identify different shapes as to identify different orientations. The use of pictures of mono-oriented objects would clarify the basis for the subjects' responses, since several objects could be used as stimuli, and, in any case, it is unlikely that a real object would be identified as a different shape in a different orientation.

(Received for publication December 10, 1979; revision accepted December 10, 1980.) 